\title{
Conformational decoupling in acid-sensing ion channels uncovers mechanism and stoichiometry of PcTx1-mediated inhibition
}

Christian B. Borg\#, Stephanie A. Heusser\#, Janne M. Colding, Stephan A. Pless*

Center for Biopharmaceuticals, Department of Drug Design and Pharmacology, University of Copenhagen, 2100 Copenhagen, Denmark

\footnotetext{
"Contributed equally

* Corresponding author:

Stephan Pless

Center for Biopharmaceuticals

Department of Drug Design and Pharmacology

University of Copenhagen

Jagtvej 160, 2100 Copenhagen, Denmark

Mail: stephan.pless@sund.ku.dk
} 


\begin{abstract}
Acid-sensing ion channels (ASICS) are trimeric proton-gated cation channels involved in fast synaptic transmission. Pharmacological inhibition of ASIC1a reduces neurotoxicity and stroke infarct volumes, with the cysteine knot toxin Psalmotoxin-1 (PcTx1) being one of the most potent and selective inhibitors. PcTx1 binds at the subunit interface in the extracellular domain (ECD), but the mechanism and the conformational consequences of the interaction, as well as the number of toxin molecules required for inhibition remain unknown. Here we use voltage-clamp fluorometry and subunit concatenation to decipher the mechanism and stoichiometry of PcTx1 inhibition. We propose PcTx1 to have at least three binding modes: 'Loose', 'Global' and 'ECDonly'. The loose and ECDonly modes are decoupled from the pore, with the latter inducing a long-lived ECD conformation that reduces the activity of an endogenous neuropeptide. This long-lived conformational state is proton-dependent and can be destabilized by the F350L mutation. Lastly, our concatemeric channel constructs reveal that disruption of a single PcTx1 binding site is sufficient to destabilize the toxin-induced conformation, while functional inhibition is not impaired until two or more binding sites are mutated. We therefore decipher the mechanism of PcTx1 inhibition and uncover a prolonged conformational change with possible pharmacological implications.
\end{abstract}




\section{Introduction}

Acid-sensing ion channels (ASICS) are trimeric proton-gated cation channels expressed throughout the central and peripheral nervous system (Gründer 2020). The extracellular domains (ECDs) of ASICs sense pH changes, allowing cations to permeate through the channel pore comprised of the transmembrane domain (TMD) (Figure 1A). Research over the last two decades has shown that this $\mathrm{pH}$-activated current underlies various physiological processes, such as fast synaptic transmission (Du, Reznikov et al. 2014), learning and memory (Wemmie, Chen et al. 2002). Due to recent reports of ASIC1a peptide-induced inhibition exhibiting neuroprotective effects, these trimeric channels have emerged as a potential drug target in neurological diseases, including protection from acidosis-induced neurotoxicity and reduction of infarct volume in mouse models (Xiong, Zhu et al. 2004, Wemmie, Taugher et al. 2013, Chassagnon, McCarthy et al. 2017, Qiang, Dong et al. 2018, Gründer 2020).

ASICs exhibit a rich pharmacology with complex molecular details. Modulators can affect activation, steadystate desensitization (SSD) - a state reached at sub-activating proton concentrations that reduces subsequent activation-but also metabotropic downstream signalling (Kellenberger and Schild 2015, Wang, Wang et al. 2015, Wang, Liu et al. 2020). Venom-derived peptides are currently the most selective and potent modulators of ASIC1 and therefore serve as valuable tools to gain deeper insights into the mechanisms of ASIC modulation (Cristofori-Armstrong and Rash 2017). Psalmotoxin-1 (PcTx1) is a 40-residue cysteine knot peptide toxin extracted from tarantula venom (Escoubas, De Weille et al. 2000). It acts as a potent gating modifier on most ASIC1a-containing channels (Chen, Kalbacher et al. 2005, Chen, Kalbacher et al. 2006) and shifts activation and SSD curves to more alkaline values, thereby effectively inhibiting the channel in the low nanomolar range at neutral pH (Cristofori-Armstrong and Rash 2017). Mutation of hASIC1a residue F352 (F350 in mASIC1a) to either alanine or leucine, and isoforms that naturally carry these residues, show significantly attenuated PcTx1 effects (Sherwood, Franke et al. 2009, Sherwood, Lee et al. 2011, Saez, Deplazes et al. 2015, Joeres, Augustinowski et al. 2016, Cristofori-Armstrong, Saez et al. 2019). Additional species- and state-dependent factors can greatly influence the functional outcome of PcTx1 modulation (Sherwood, Lee et al. 2011, Cristofori-Armstrong, Saez et al. 2019), yet little is known about the conformational consequences of the interaction in different functional states and under varying $\mathrm{pH}$ conditions. This is relevant, since long-lived conformational changes could, for example, affect the activity of endogenous ASIC1a modulators. Lastly, structural studies have shown that PCTX1 binds to the subunit interfaces of CASIC1, forming contact points with the primary and complementary side of the acidic pocket (Dawson, Benz et al. 2012, Baconguis, Bohlen et al. 2014) (Figure 1A), but it remains unclear how many toxin molecules are required to obtain functional channel modulation.

Here we show that PcTX1 has at least three distinct binding modes of which at least one induces a long-lived conformational state in the ASIC1a ECD that alters the effects of the endogenous neuropeptide Big Dynorphin (BigDyn). The ECD conformational state further uncovers an unexpected decoupling from the state of the pore that has profound implications for the stoichiometry of the ASIC1a-PCTX1 interaction: while disrupting a single PcTX1 binding site in an ASIC1a trimer is sufficient to destabilize the long-lived PCTX1-induced ECD conformational state, functional inhibition of the channel is only affected when two or more PcTx1 binding 
bioRxiv preprint doi: https://doi.org/10.1101/2021.06.21.449215; this version posted August 26, 2021. The copyright holder for this preprint (which was not certified by peer review) is the author/funder. All rights reserved. No reuse allowed without permission.

sites are mutated. Collectively, we illustrate how a combination of voltage-clamp fluorometry and subunit engineering provides detailed mechanistic insights on a potentially therapeutically relevant channel-toxin interaction and demonstrate how these findings affect fundamental pharmacological properties of the channel. 


\section{Results}

\section{PcTX1 induces long-lived conformational changes in ASIC1a}

First, we sought to establish the functional consequences of PcTx1 binding to mASIC1a. In line with previous findings, $30 \mathrm{nM}$ of PcTx1 led to potent alkaline shifts in both the activation curve $\left(\Delta \mathrm{pH}_{50}\right.$ Act $>0.5 \mathrm{pH}$ units at pH 7.9; Figure $1 B$, D; Table S1) and the SSD of ASIC1a WT expressed in Xenopus laevis oocytes $\left(\Delta \mathrm{pH}_{50} \mathrm{SSD}_{2} \approx 0.4 \mathrm{pH}\right.$ units; Figure 1C, D; Table S1). While this electrophysiological characterisation provides a detailed view of how PCTX1 affects channel function, as assessed by the state of the pore, it is unable to capture the extent and time course of possible conformational consequences caused by the toxin-channel interaction in the ECD of ASIC1a. In other words, it remained unclear if potential toxin-induced conformational changes extend across the entire ECD and how long they persist. We therefore introduced a cysteine residue into the MASIC1a ECD at amino acid position 80 or 105 (Figure $1 \mathrm{~A}$ ) and labelled it with the environmentally sensitive AlexaFluor488 dye via a maleimide linker (Vullo, Bonifacio et al. 2017). This allowed us to monitor changes in the fluorescence signal upon exposure to different proton or toxin concentrations and thus provide a proxy for ECD conformational changes at two spatially distant positions. For AlexaFluor488-labelled K105C mASIC1a (K105C*), application of pH 5.5 resulted in an inward current and a simultaneous upward deflection of the fluorescence signal (Figure $1 \mathrm{E})$. The $\mathrm{pH}$ dependence of the fluorescence signal for $\mathrm{K} 105 \mathrm{C} *^{*}$ was similar to the $\mathrm{pH}_{50} \mathrm{SSD}_{\text {, }}$ indicating that the conformational changes tracked by fluorescent labelling could reflect a transition related to a desensitized state (Figure S1A, B, Table S3; note that pH changes between 7.4 and 9.0 did not lead to any fluorescence changes (Borg, Braun et al. 2020)). Application of $300 \mathrm{nM} \mathrm{PcTx1}$ at pH 7.4 resulted in slow upward deflection of the fluorescence (Figure 1E, left panel; Table S4). During a 3 min washout with pH 7.4 solution, the fluorescence signal displayed only a modest decrease, while post-PcTx1 exposure to $\mathrm{pH} 8.4$ led to an immediate return of fluorescence to baseline level. However, the fluorescence increased as soon as the $\mathrm{pH}$ was switched back to 7.4, suggesting the presence of a conformational change that is sustained at $\mathrm{pH} 7.4$. We observed similar effects with two other $\mathrm{pH}$ application protocols after PcTx1 exposure (Figure 1E, middle panel, Figure 1F and Figure S1C, E, Table S6).

To rule out a direct interaction between the fluorophore and PcTx1 or the possibility of a local conformational change, we also labelled the channel in position V80C of the palm domain (V80C*, Figure $1 \mathrm{~A}$ ) and characterized the $\mathrm{pH}$ and PcTx1 responses. Here, the direction of the fluorescence signal upon activation and desensitization was inverted (Figure 1G), but application and washout of $300 \mathrm{nM} \mathrm{PcTx1}$ at $0.3 \mathrm{pH}$ units more alkaline than $\mathrm{pH}_{50}$ of the fluorescence signal in V80C* resulted in qualitatively similar effects as observed for K105C* (Figure 1G, H; S1D, F). In light of the data obtained from K105C* and V80C*, we considered it implausible that the fluorescence changes are the consequence of a direct interaction between the dye and the peptide, but rather result from a global conformational change of the ECD upon PCTX1 binding. We conclude that PCTX1 binding induces a long-lived conformational change and that this conformational alteration is not necessarily coupled to the channel pore. 


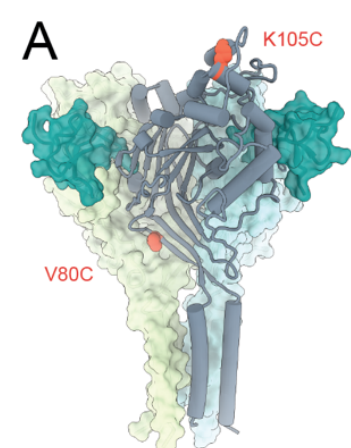

B

C
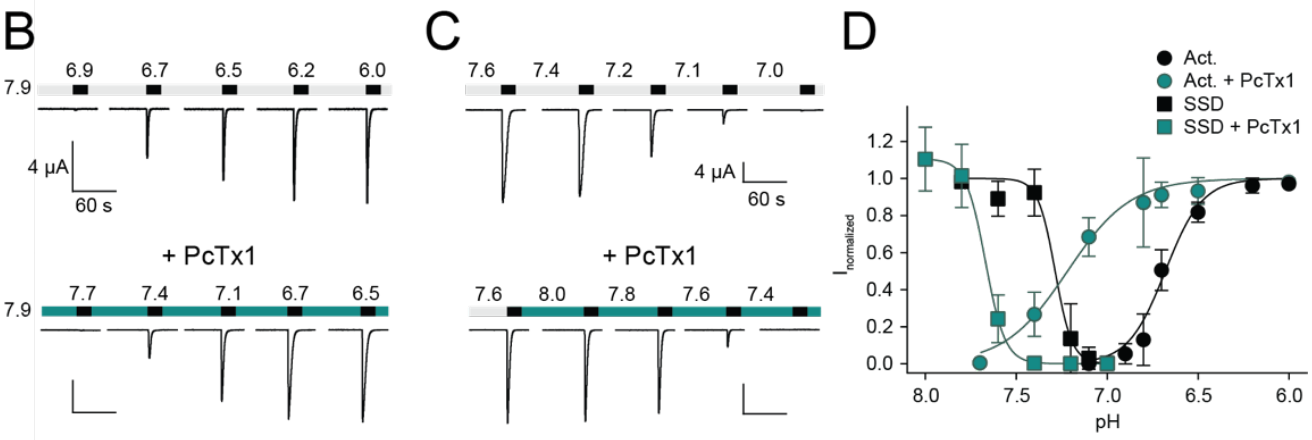

$\mathrm{E}$
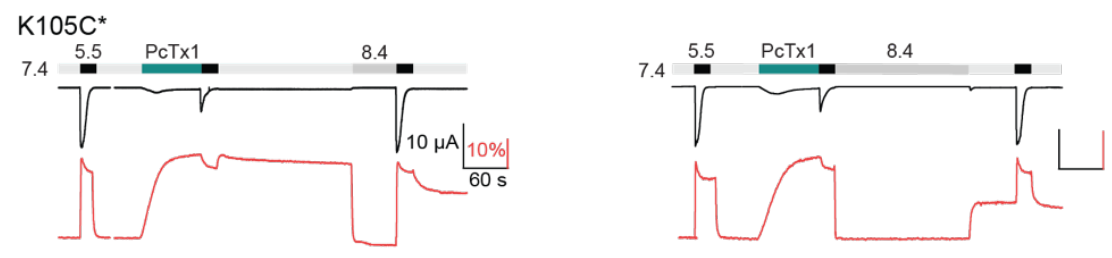

$\mathrm{F}$

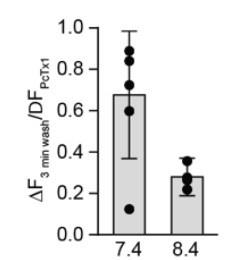

G
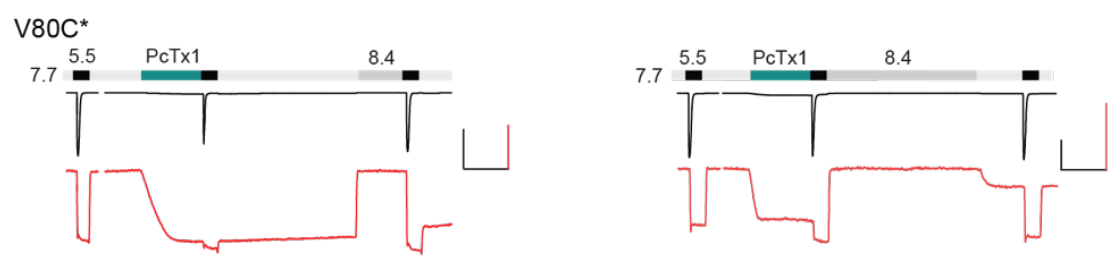

$\mathrm{H}$

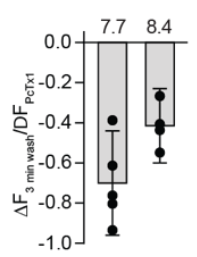

Figure 1. PcTx1 induces a distinct long-lived conformational state in the ASIC1a ECD. (A) Structural overview (PDB ID 4FZO) of chicken ASIC1 with positions V80 and K105, which were substituted for cysteine and used for channel labelling, highlighted in red. PcTx1 (teal) binds to the subunit interfaces. (B) Representative two-electrode voltage clamp (TEVC) traces recorded from X. laevis oocytes of WT ASIC1a showing pH sensitivity of activation in absence (upper panel) and presence (lower panel) of $30 \mathrm{nM}$ PcTX1, added in the resting solution (pH 7.9) (C) Same as in (B) but for steady-state desensitization (SSD). PcTx1 was applied to solutions of decreasing pH in between application of activating pH 5.6 solution. (D) Concentration-response relationship of WT ASIC1a activation and SSD in absence and presence of $30 \mathrm{nM}$ PCTX1 retrieved form experiments shown in (B) and (C). (E) Representative traces of voltage-clamp fluorometry (VCF) recordings of K105C* with the current in black and the fluorescence in red. PcTx1 (300 nM) was washed off for $3 \mathrm{~min}$ using pH 7.4 (left) or pH 8.4 (right). (F) Quantitative analysis of the fluorescence signal at the end of the 3 min washout protocols shown in B relative to the fluorescence observed upon PCTx1 application. (G) Representative trace of a VCF recording of V80C* equivalent to the ones shown in (E). (H) Same as in (F) but for V80C*F350L. Scale bars are $60 \mathrm{~s}$ (black horizontal), $4 \mu \mathrm{A}(\mathrm{B}-\mathrm{C})$ and $10 \mu \mathrm{A}$ (E and G) (black vertical), and $10 \%$ (red, $\mathrm{E}$ and $\mathrm{G}$ only). Data in $\mathrm{D}, \mathrm{F}$ and $\mathrm{H}$ are presented as mean $+95 \mathrm{Cl} ; \mathrm{n}=3-18$ for individual data points in $\mathrm{D}$.

\section{PcTX1 has three distinct binding modes and pre-exposure can alter channel pharmacology}

Next, we sought to further probe the stability, as well as the functional and pharmacological implications of this long-lived conformational state. Strikingly, we found that repeated exposure to $\mathrm{pH} 5.5$ led to a gradual recovery of the current amplitude, while the fluorescence signal remained effectively unchanged (Figure 2A). This experiment emphasized a clear decoupling between the conformational state of the ECD and the functional consequences at the level of the pore: while the pore slowly recovered from its inhibited state and regained its ability to conduct ions, the ECD conformation remained essentially unaltered, even after minutes of washout.

Together, our data raised the intriguing possibility that PcTx1 may have different binding modes. To address this notion directly, we devised a series of experiments in which PcTx1 was applied during a pH 8.0-induced closed state before switching to different $\mathrm{pH}$ regimes. We had already established that 
application of PcTX1 at pH 7.4 can elicit a long-lived conformational state that persists beyond the washout of the toxin and remains stable even after the current has recovered (Figure 1E, G and Figure 2A). Similarly, this conformation can be evoked if PcTx1 is applied at a pH 8.0-induced closed state immediately before channel activation (Figure 2B, left panel and Figure 2C) or by administering PcTx1 during a pH 8.0-induced closed state before exposing the now highly proton-sensitive channel to $\mathrm{pH} 7.4$, which likely results in temporary SSD upon exposure to $\mathrm{pH} 7.4$ (Figure 2B, middle panel and Figure 2C). We therefore conclude that all conditions that either temporarily activate and/or desensitize the channel can elicit a long-lived PCTX1-induced conformational state that is decoupled from the pore. In stark contrast, when a pH 8.0induced closed state is maintained before, during and after PcTx1 application, pH 7.4 is no longer able to elicit a conformational change (Figure 2B, right panel and Figure $2 \mathrm{C}$ ). This indicates PcTx 1 binding to the closed state is readily reversible and does not result in a lasting conformational change in the ECD. Based on these findings, we propose that PCTX1 has three distinct binding modes: 'Loose', a loosely bound high $\mathrm{pH}$-state that is not associated with ECD conformational changes; 'Global', a tightly bound state that is reached in the presence of PcTX1 applied at neutral/low pH and that has global effects on the functional state of the pore and conformational (ECD) consequences, and 'ECDonly', a state in which PcTx1 is tightly bound, that exists at neutral/low pH even in the absence of PcTx1 in the solution, with long-lasting conformational alterations in the ECD only (Figure 2D).

If correct, the tightly bound 'ECD only' state would be expected to affect the activity of other ECD-targeting ASIC1a modulators. To test this possibility, we turned to BigDyn, a neuropeptide that competes with PcTx1 for the binding site and induces a distinct closed state in ASIC1a (Sherwood and Askwith 2009, Borg, Braun et al. 2020). The latter is indicated by a robust and distinct downward deflection of the K105C* fluorescence signal (Figure 2E). Strikingly, we found that when BigDyn (1 $\mu \mathrm{M})$ is applied to the PcTx1induced 'ECD only' state, the neuropeptide has a much less pronounced effect on the fluorescence signal than without PcTx1 pre-exposure (Figure 2E, F, Table S5). To ensure that this effect is indeed specific to PCTX1, we conducted a control experiment in which we first applied BigDyn and then switched to PcTx1 (Figure S1G). In this case, the PcTx1-elicited fluorescence response post BigDyn exposure was unchanged compared to that observed in control cells (Figure 2F, Table S5). In summary, these findings support the notion that the tightly bound ' $E C D_{\text {only' }}$ state, although without immediate consequences for the channel pore, does have direct implications for binding of other ECD-targeting compounds. 
A

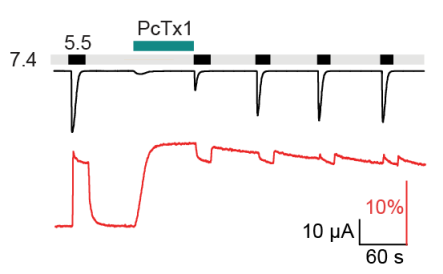

B
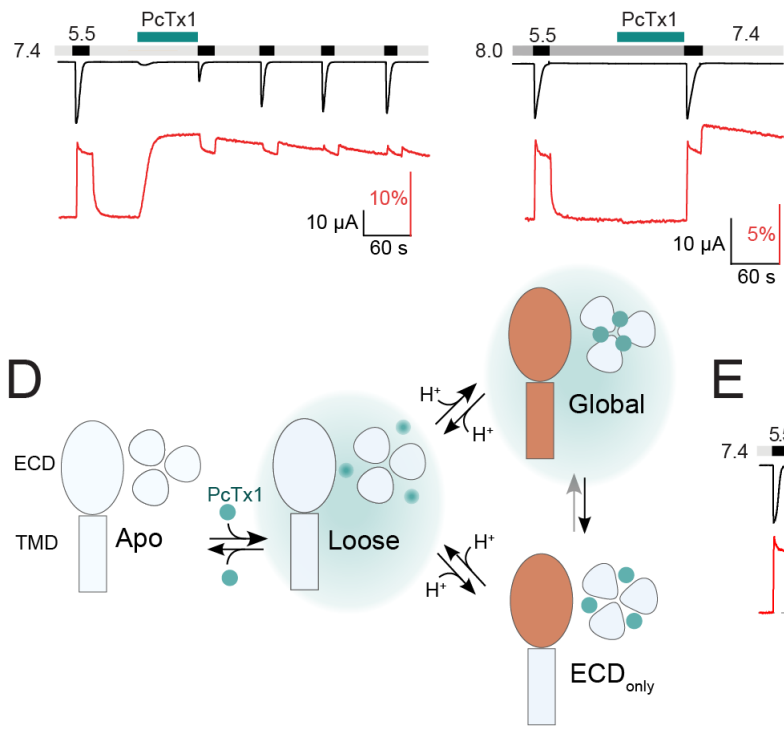
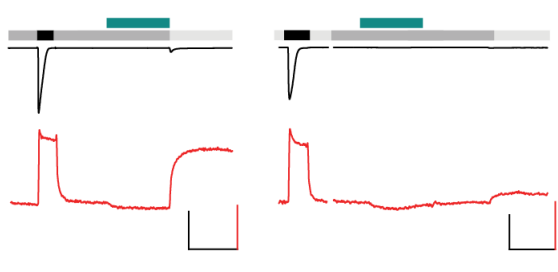

C

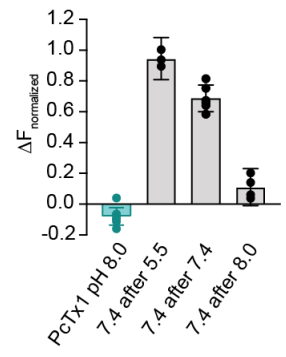

E

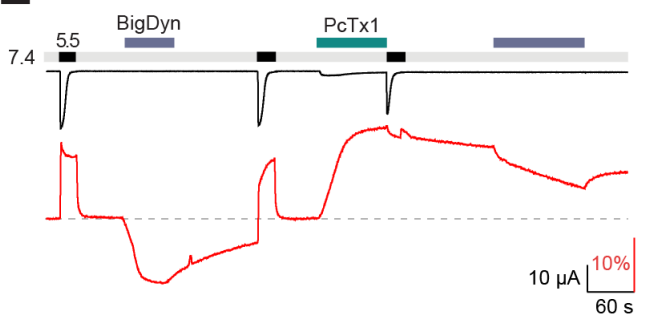

$\mathrm{F}$

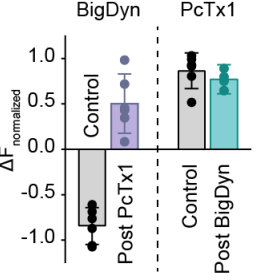

Figure 2. PcTX1 has pH-dependent binding modes and alters ASIC1a pharmacology. (A) VCF trace of K105C* showing that the channel readily returns to a functional 'apo' state (current, black trace) after application of 300 nM PcTx1 at pH 7.4, while the fluorescence change induced by PCTX1 is persistent over multiple ASIC1a activations at pH 5.5 (fluorescence, red trace). (B) VCF traces highlighting the fluorescence changes associated with application of PcTx1 at pH 8.0 with subsequent application of pH 5.5 (left), pH 7.4 (middle) and pH 8.0 (right). (C) Quantitative comparison of the fluorescence signal $60 \mathrm{~s}$ into the pH 7.4 application at the end of the experiments shown in (B) normalized to the fluorescence change induced by pH 5.5 application. (D) Schematic representation of the different pH dependent binding modes of PcTx1: A 'Loose' closed state at high pH, a 'Global' state that exists at neutral/low pH in the presence of PCTX1 and leads to conformational rearrangements in both ASIC1a ECD and pore (indicated in orange), and an 'ECD only' state in which the conformational rearrangements are only found in the ECD and that exists at neutral/low pH even when PxTx1 is absent in the extracellular solution. Teal background shading indicates the presence of PcTx1 in the extracellular solution. Transitions between the binding modes that are explicitly shown in this work are indicated in full opacity. (E) VCF trace of K105C* exposed to pH 5.5, followed by a $60 \mathrm{~s}$ BigDyn (1 $\mu \mathrm{M}$ ) application (purple bar), with subsequent washout and activation. BigDyn is then applied again after the 'ECD only' state is evoked through PcTx1 (300 nM) application, this time only resulting in a slow decrease in the fluorescence signal. (F) Quantitative comparison of the fluorescence change induced by a $60 \mathrm{~s}$ BigDyn application to the 'apo' (Control) and to the PcTx1-induced 'ECDonly' state (Post PcTx1), normalized to the signal induced by pH 5.5; and of the fluorescence signal induced by PcTx1 at pH 7.4 (Control) and BigDyn pre-application (Post BigDyn), respectively. Scale bars are $60 \mathrm{~s}$ (black horizontal), $10 \mu \mathrm{A}$ (black vertical), and 10\% (A and E) or $5 \%$ (B) (red, A, B and E only). Data in $\mathrm{C}$ and $\mathrm{F}$ are presented as mean $+95 \mathrm{Cl}$.

\section{F350L diminishes PCTX1 sensitivity and stability of toxin-induced conformation}

Next, we wanted to establish how PcTx1 sensitivity and binding modes change in the F350L mutation that renders the channel virtually insensitive to the toxin (Sherwood, Franke et al. 2009, Saez, Deplazes et al. 2015). We thus compared the PcTx1 sensitivity of the F350L mutant to that of WT mASIC1a (Figure 3A) at a pH that would neither activate nor steady-state desensitize the channels in the absence of the toxin ( $\mathrm{pH} 7.9$ for WT; pH 7.6 for F350L). Consistent with previous findings (Sherwood, Franke et al. 2009), introducing the F350L mutation reduced the $\mathrm{pH}$ sensitivity of activation and SSD, and the presence of $30 \mathrm{nM}$ PcTx1 only led to a slight shift in $\mathrm{pH}_{50}$ SSD, with no discernible effect on channel activation (Figure 3B, C; Table S1). The inhibitory effect of PcTx1 under physiological pH (7.4) was still concentration dependent but dramatically decreased in the F350L variant compared to WT (IC 50 of around $1 \mu \mathrm{M}$ vs. IC $\mathrm{C}_{50}$ of around $0.6 \mathrm{nM}$; Figure 3D, E; Table S2). 
Introduction of the F350L mutation therefore strongly reduced the gating-modifying properties of PcTX1 and diminished its inhibitory effect by three orders of magnitude.

In contrast to the K105C* variant, we found that application of $300 \mathrm{nM} \mathrm{PCT \times 1} \mathrm{to} \mathrm{K105C*F350L} \mathrm{at} \mathrm{pH} 7.4$ did not elicit any fluorescence changes (Figure 3F, Table S4). Notably, the fluorescence response curve of the K105C*F350L variant is about $0.1 \mathrm{pH}$ units shifted towards more acidic pHs compared to K105C* (Figure S1B, Table S3). Taking this change into account, we applied PcTx1 at pH 7.3. Under these conditions, PcTx1 induced a similar slow-onset fluorescence signal as seen for K105C*, albeit slightly less pronounced, while the functional insensitivity of the F350L variant to PCTX1 remained (Figure 3G, left; Table S4) indicating that the channel can still enter the 'ECD only' state while the 'global' binding mode is unfavourable. A striking difference was apparent in the washout: unlike in K105C*, the fluorescence signal of K105C*F350L readily returned to a level indicative of a closed apo state in the first 1-2 min of $\mathrm{pH} 7.4$ washout, suggesting that the longevity of the 'ECDonly' state is destabilized (Figure 3G, right; Table S6). Again, these observations were mirrored by the V80C*F350L variant (Figure $3 \mathrm{H}$ ), thereby emphasising the robust and global nature of the observed conformational changes. Together, these data suggest that the functional PCTX1 insensitivity of the F350L variant primarily originates from a destabilization of the PcTx1-induced conformational states ('Global' and 'ECD only') rather than solely from impaired binding. 
A

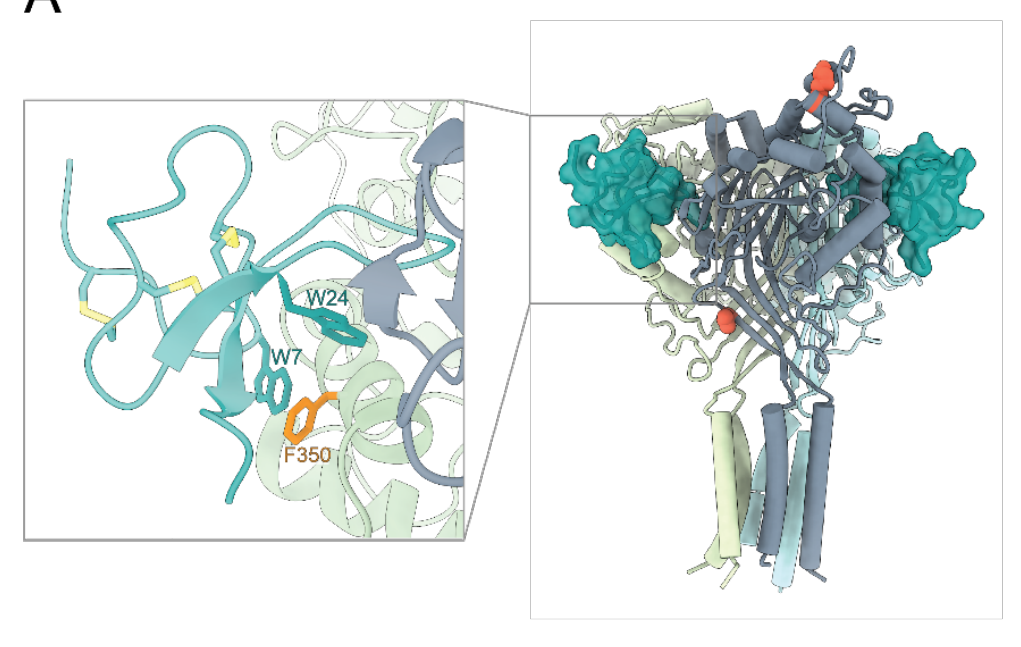

D

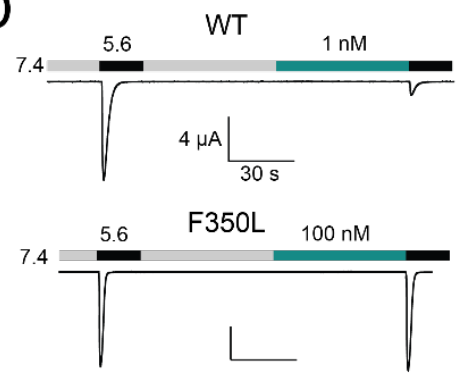

E

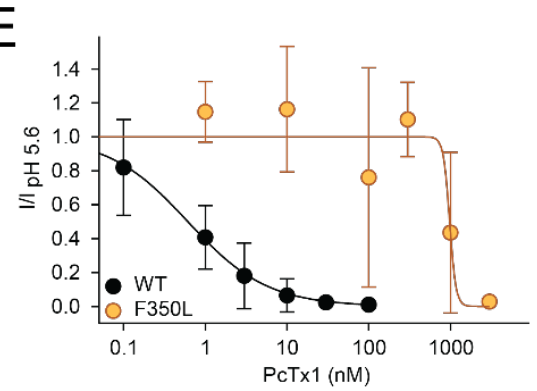

B
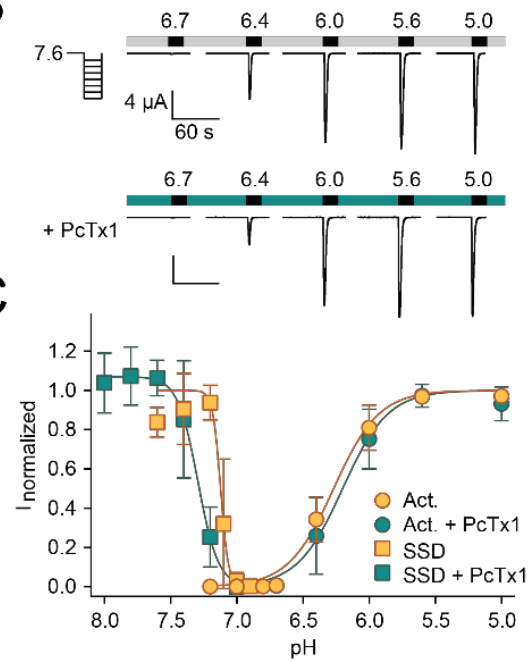

$\mathrm{F}$

G K105C*F350L
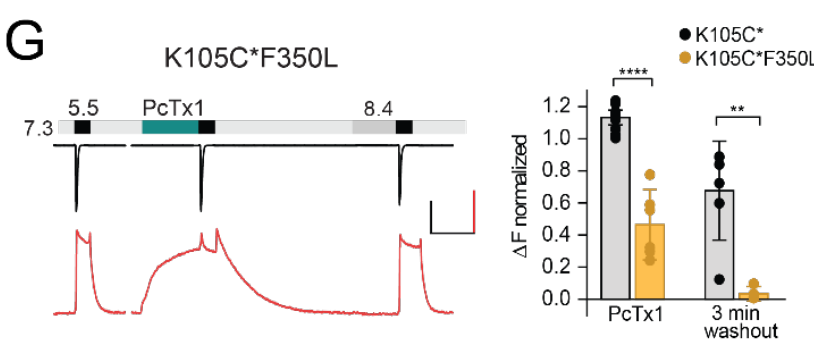

$\mathrm{H}$

V80C*F350L

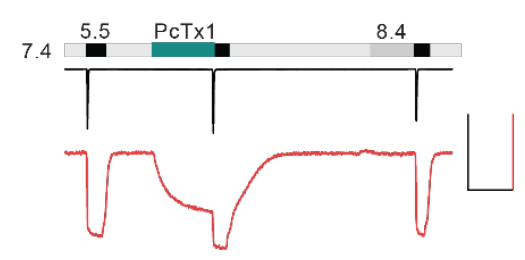

$\mathrm{K} 105 \mathrm{C}^{*} \mathrm{~F} 350 \mathrm{~L}$

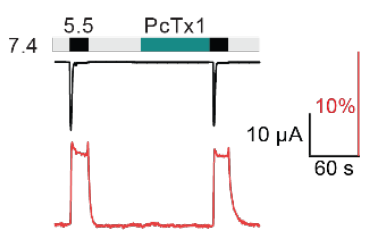

The F350L mutation diminishes PCTX1 sensitivity by destabilizing the toxin-induced conformation. (A) Model of the co-crystal structure of CASIC1a and PCTX1 (teal) binding to the extracellular domain (PDBID 4FZO). Inset shows a close up of the interaction site at the acidic pocket, including ASIC1a residue F350 (orange). (B) Representative TEVC trace showing mASIC1a F350L pH activation in the absence (top) and presence (bottom) of $30 \mathrm{nM}$ PCTx1. (C) Activation and SSD curve of F350L mASIC1a without (orange) and with (teal) PcTx1 ( $n=5-12)$. (D) TEVC traces showing the effect of 1 nM PcTx1 on mASIC1a WT (top) and $100 \mathrm{nM} \mathrm{PcTx1} \mathrm{on} \mathrm{F350L} \mathrm{(bottom)} \mathrm{applied} \mathrm{at} \mathrm{pH}$ 7.4. (E) PcTx1 concentration-response curve at $\mathrm{pH} 7.4$ using the protocol shown in (D) $(n=4-14)$. (F) Representative VCF trace of the K105C*F350L mutant showing application of $300 \mathrm{nM} \mathrm{PCTx1} \mathrm{at} \mathrm{pH} \mathrm{7.4.} \mathrm{(G)} \mathrm{Left:} \mathrm{Representative} \mathrm{VCF} \mathrm{trace} \mathrm{of} \mathrm{the} \mathrm{K105C*F350L} \mathrm{mutant}$ showing application of $300 \mathrm{nM}$ PcTx1 at pH 7.3. Right: Comparison of the fluorescence change upon PcTx1 application and after a 3 min washout between K105C* and K150C*F350L (H) Left: Representative trace of a VCF recording of V80C*F350L equivalent to the one shown in G. Right: Same analysis as in G but compared between V80C* and V80C*F350L. Scale bars are $60 \mathrm{~s}$ (black horizontal), 4 $\mu \mathrm{A}$ ( $\mathrm{B}$ and $\mathrm{D}$ only) and $10 \mu \mathrm{A}$ (black vertical), and 10\% (red) (F-H). Data in $\mathrm{C}, \mathrm{E}, \mathrm{G}$ and $\mathrm{H}$ are presented as mean $+95 \mathrm{Cl}$.

\section{Contribution of individual F350L subunits to PcTX1 inhibition}

The drastic reduction in PcTx1 sensitivity and notable destabilization of the PcTx1-induced conformational state through the F350L mutation provided a unique opportunity to determine the stoichiometric requirements for functional PCTX1 modulation, as well as the contribution of individual F350L-containing ASIC1a subunits to PcTX1 sensitivity. Conventional approaches are inapt to answer these fundamental questions, as it is technically 
challenging to introduce targeted mutation in a single subunit within a homotrimer, and co-expression of nonconcatenated WT and mutant subunits can lead to undesired, heterogeneous subunit assemblies precluding accurate interpretation of functional data. The use of concatemeric constructs helps to overcome these limitations. By fusing cDNAs of subunits of interest in the desired order, this approach predetermines the subunit composition and assembly of channels, allowing us to directly address questions such as the number of PCTX1 binding sites needed for functional inhibition. Importantly, the trimeric assembly of ASICs has proven to be highly amenable to this approach (Joeres, Augustinowski et al. 2016, Lynagh, Flood et al. 2017, Wu, Chen et al. 2019).

We therefore designed concatemeric ASIC1a constructs of triple WT subunits (WT/WT/WT) and constructs carrying the F350L substitution in one, two, or all three subunits (F350L/F350L/F350L), using a previously validated construct design (Figure 4A; S2) (Lynagh, Flood et al. 2017). The pH-dependence of the WT/WT/WT and the F350L/F350L/F350L concatemer constructs showed similar pH sensitivities compared to WT and F350L, respectively (Figure 4B-E; Table S7). The number of F350L subunits determined the pH dependence of concatemeric ASIC1a constructs but was not dependent on the relative positioning of the mutation within the concatemeric ASIC1a construct (Figure 4B-E; S3A, B; Table S7). These results provided a robust foundation to assess the effect of one, two or three F350L-bearing subunits on PcTx1 inhibition. 
A

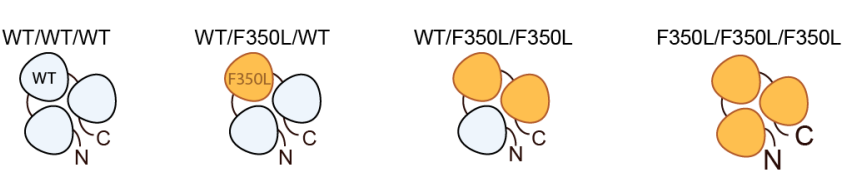

WT/WT/WT

WT/F350L/WT

WT/F350L/F350L

F350L/F350L/F350L
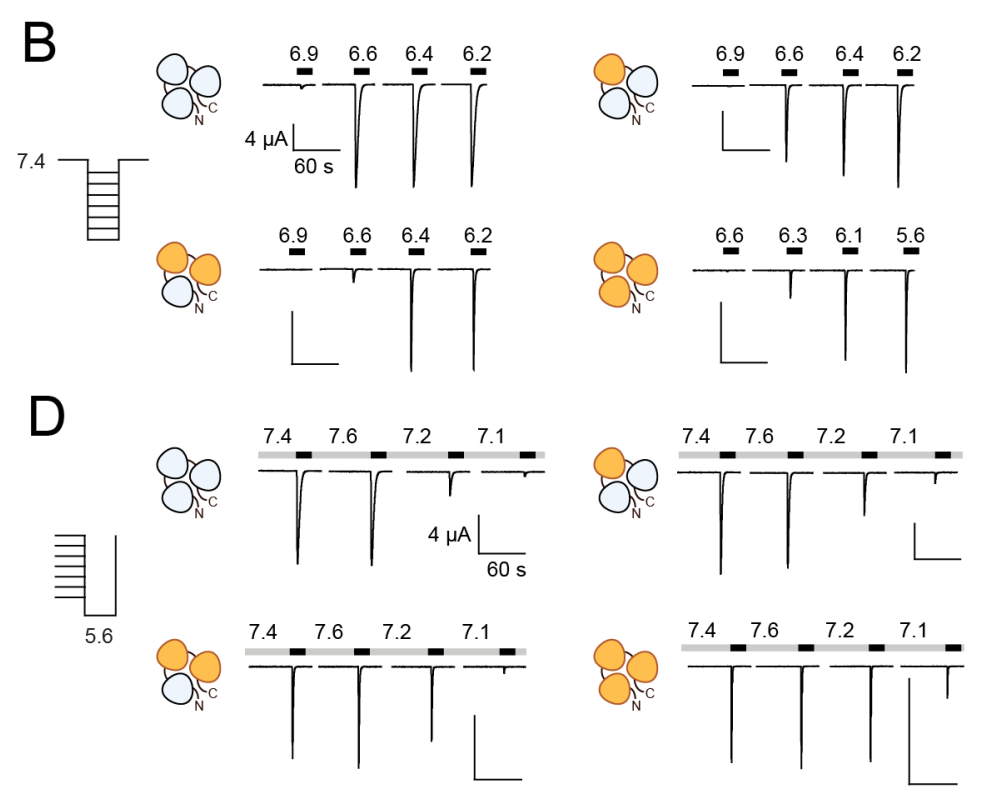

F
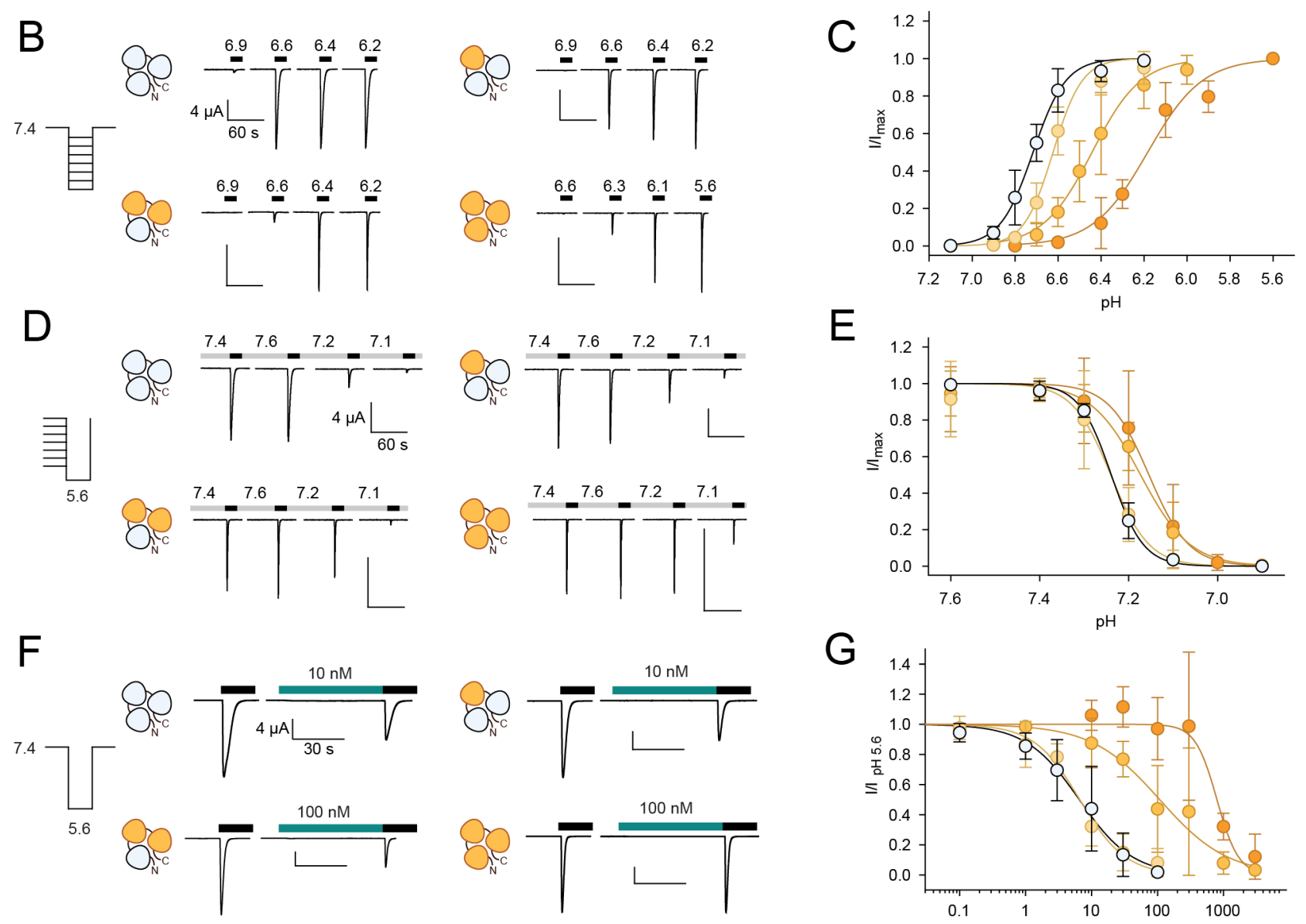

$\mathrm{E}$
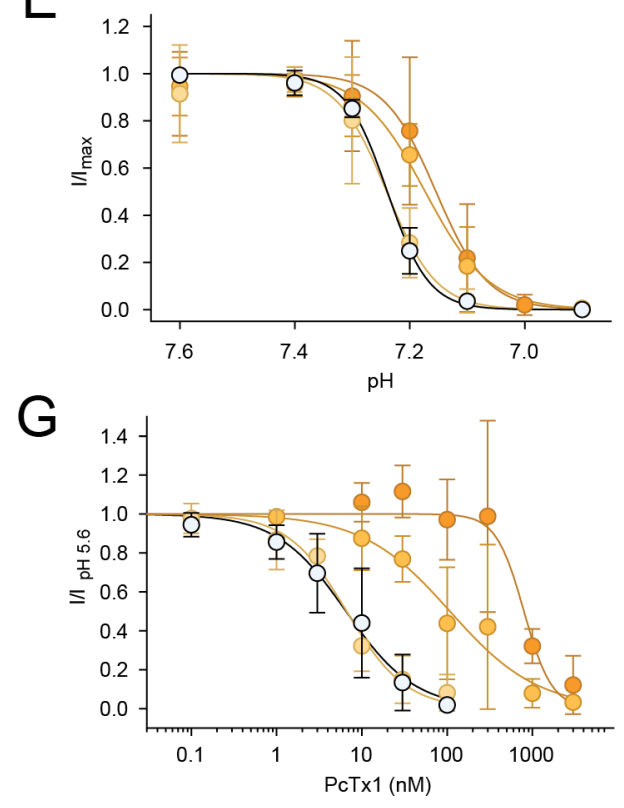

Figure 4. In concatemeric constructs, two WT subunits are sufficient for WT-like PcTX1 inhibition. (A) Schematic overview of the concatemeric constructs containing the F350L mutation (orange) in none, one, two or all three subunits. (B) Representative TEVC trace of activation. (C) Activation curve from recordings shown in $B$ for the four different concatemeric constructs ( $n=7-13)$ (D) Representative TEVC trace of SSD (E) SSD profiles from recordings shown in $D(n=4-11)$. (F) Representative TEVC trace of concentration dependent PcTx1 inhibition at pH 7.4. (G) PcTx1 concentration-response curves from data shown in $F(n=4-11)$. Data points in $E-G$ represent mean and $95 \mathrm{Cl}$. All scale bars are $4 \mu \mathrm{A}$ and $60 \mathrm{~s}(\mathrm{~B}$ and $\mathrm{D})$ or $30 \mathrm{~s}(\mathrm{~F})$, respectively.

For the WT/WT/WT concatemer, pH 5.6-induced currents were inhibited by PcTx1 with an apparent IC 50 of around $6 \mathrm{nM}$ at pH 7.4, compared to an $\mathrm{IC}_{50}$ of $0.6 \mathrm{nM}$ for WT ASIC1a (Figure 4F, G; Table S2). For the F350L/F350L/F350L concatemer, PcTx1 inhibited pH 5.6-induced currents with an apparent IC $_{50}$ of around $800 \mathrm{nM}$, similar to the trimeric F350L ASIC1a mutant (Figure 3E; 4F, G; Table S2). This meant that despite the lower PcTX1 sensitivity of the WT/WT/WT concatenated construct, the substantial decrease in PcTX1 sensitivity caused by the F350L mutation was maintained in the F350L/F350L/F350L construct. For concatenated constructs containing two F350L-bearing subunits (WT/F350L/F350L), pH 5.6-induced currents were inhibited by PcTx1 with an apparent IC 50 around 100 nM (Figure 4F, G; S3C, Table S2). This represented a 17-fold increase in apparent $I_{50}$ compared to the WT/WT/WT concatemer and an 8-fold decrease compared to the 
F350L/F350L/F350L concatemer. Remarkably, the concatemer with only a single F350L-containing subunit (WT/F350L/WT) closely matched the PcTx1 inhibition profile observed for of the WT/WT/WT concatemer. (Figure 4F, G; S3C; Table S2).

Taken together, we demonstrate that application of PcTx1 at pH 7.4 maintains the WT-like inhibitory effect on pH 5.6 activation even if one out of three subunits contains the F350L mutation. We conclude that two WT subunits per mASIC1a trimer are sufficient to observe WT-like functional inhibition by PcTx1.

\section{PcTx1-induced conformational changes in concatemeric channels}

We next wanted to test if a stepwise introduction of F350L-bearing subunits would result in a difference in the stability of the PCTX1-induced ECD conformational states observed in VCF experiments. To this end, we introduced the $\mathrm{K} 105 \mathrm{C}$ mutation in all three subunits for AlexaFluor488-labelling, while increasing the number of F350L-containing subunits from one to two and three, respectively. We refer to subunits with WT background but labelled at $\mathrm{K} 105 \mathrm{C}$ as WT*, while subunits labelled at $\mathrm{K} 105 \mathrm{C}$ and containing the $\mathrm{F} 350 \mathrm{~L}$ mutation are designated F350L*. First, we established the $\mathrm{pH}$ dependence of the fluorescence signal. Overall, the $\mathrm{pH}_{50}$ of the fluorescence and the $\mathrm{pH}_{50}$ SSD matched in all constructs and were in a similar range as the nonconcatenated channels (Figure S4, Table S3). PcTx1 (300 nM) was applied 0.3 pH units above the measured

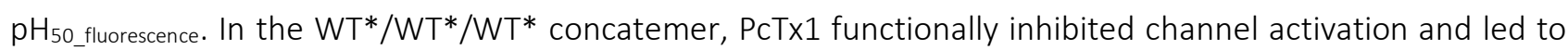
the characteristic increase in fluorescence maintained even after removal of PcTx1 from the buffer, and this was comparable to what we observed in the K105C* trimer (Figure 1E; 5A, D, E; Table S4, S6).

In WT*/F350L*/WT*concatemers, PCTX1 still had an inhibitory effect on activation, in line with the results from non-labelled constructs, and produced a robust fluorescence response (Figure 1E, D; Table S4). In this construct, a marked gradual decrease of the fluorescence signal during the 3 min washout at pH 7.4 was noticeable (Figure 5B, E; Table S6). In constructs with two F350L-containing subunits, the response of the fluorescence signal to PcTx1 was further reduced, and the fluorescence signal returned to baseline levels (indicative of a closed apo state) in the first 1-2 min of the pH 7.4 washout (Figure 5C-E; Table S4, S6), reminiscent of the PCTX1 effect on the non-concatemeric K105C*/F350L channel. No reliable changes in fluorescence could be detected for the F350L*/F350L*/F350L*, likely due to low expression. 

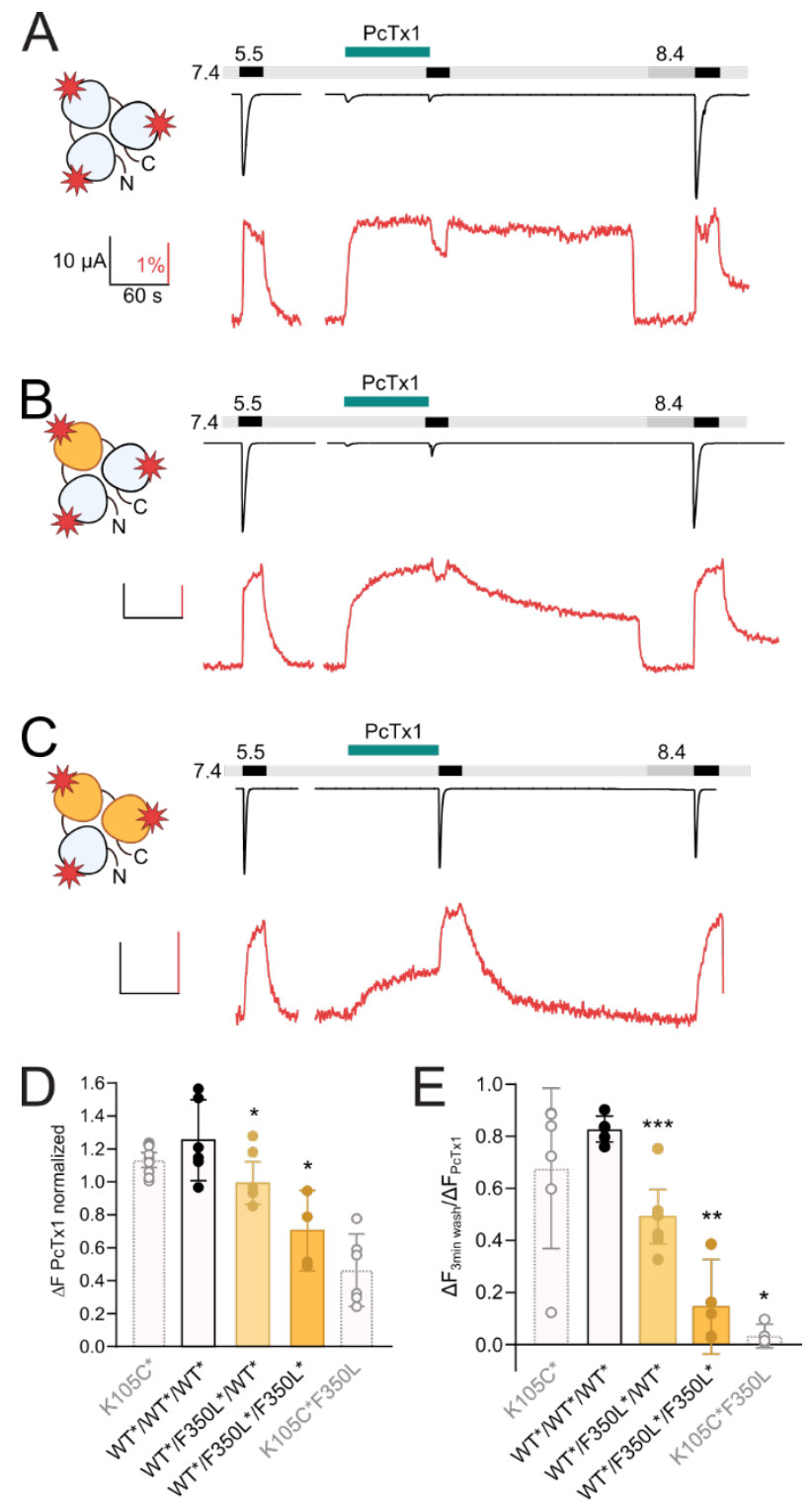

Figure 5. One F350L subunit is sufficient to destabilize the PCTx1-induced ECD conformation. (A) Representative VCF trace of 300 nM PcTx1 application to a concatemeric construct labelled at K105C* in all three subunits (red star) and subsequent washout for 3 min with pH 7.4 and 40 s pH 8.4. (B) Same as in A but one subunit carries a F350L mutation. (C) Same as in A but with two subunits carrying a F350L mutation. (D) Comparison of the PCTX1-induced change in the fluorescence signal between the different concatemeric constructs shown in A-C. Results from non-concatenated channels are indicated for comparison (shown in light grey). (E) Comparison of the fluorescence intensity after a 3 min washout relative to the intensity upon PcTx1 application. Results from non-concatenated channels are indicated for comparison (shown in light grey). All scale bars represent $10 \mu \mathrm{A}$ (black vertical), $60 \mathrm{~s}$ (black horizontal), $1 \%$ (red). In D and E, errors are $95 \mathrm{Cl}$, unpaired t-Mann-Whitney test to neighbouring bar on the left, ${ }^{*} \mathrm{P}<0.05$, $* * P<0.005, * * * P<0.0005$.

In summary, our VCF experiments demonstrate that in contrast to the functional modulation, the presence of a single F350L-containing subunit is sufficient to destabilize the conformational effect introduced by PcTx1, further emphasizing that PCTX1 can elicit distinct responses in the ECD and the pore of ASIC1a (Figure 6). 

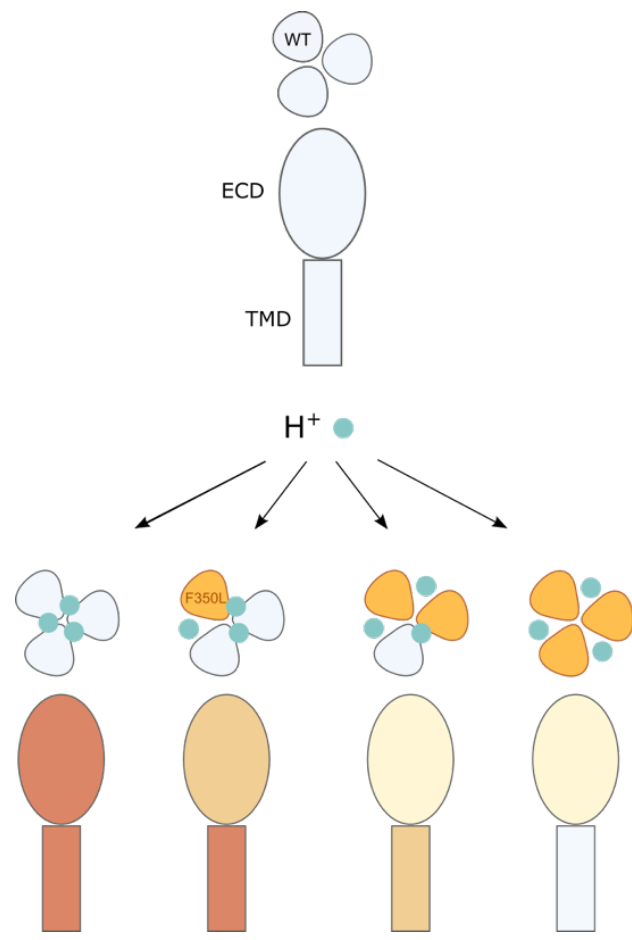

Figure 6. Illustration of the conformational and functional stoichiometry of PcTx1 binding. Schematic representation of a sideview of ASIC1a ECD and TMD and top view of the three subunits and consequences of PcTx1 (teal) binding at neutral/low pH (as in Figure 2D) and with the F350L mutation (orange) in 0-3 subunits. The side view colouring shows the decreasing stability of the PcTx1-induced 'ECD only' conformation with increasing number of F350L containing subunits, and the decreasing inhibitory effect on the pore. In channels with a single F350L subunit, only the PcTx1-induced conformational state of the ECD is affected, while the TMD behaves WTlike. 


\section{Discussion}

PCTX1 is a widely used ASIC1a modulator in in vitro and in vivo studies, yet the molecular underpinnings of binding, resulting conformational changes and stoichiometric requirements remained enigmatic. Here we employ voltage-clamp fluorometry and concatemeric ASIC1a constructs that carry a virtually PcTx1-insensitive mutation in one, two, or all three subunits to establish the stoichiometry of the ASIC1a-PcTx1 interaction. We establish three modes of action for PcTX1 and observe that at least one of them induces a ECD conformational change, which leaves the state of the pore unaffected but impacts binding of an endogenous peptide modulator. Collectively, our work provides mechanistic insight into the ASIC1a-PcTx1 interaction and emphasises the potential of protein engineering approaches for detailed studies of protein-peptide interactions.

\section{Origin of PCTX1-induced conformational changes}

VCF experiments on K105C* and V80C* showed slow-onset PcTX1-induced fluorescence changes, indicating changes in the local environment of the fluorophore attached to the finger or palm domain, respectively. As outlined above, we deem it unlikely that the fluorescence changes are brought on by a direct interaction of PcTX1 with the fluorophore, as the fluorescence signal follows opposite directions depending on the place of labelling. This leaves the question if the conformational rearrangements reflect a transition into a different functional state or are related to more local PcTx1-induced conformational changes. The phenotype of the PCTX1-induced fluorescence change resembles the one observed for SSD (Figure 1E, G and Figure S1A), and we observe good agreement between the $\mathrm{pH}_{50}$ of the fluorescence with the $\mathrm{pH}_{50}$ SSD in all variants tested here (Figure S1B, S4; Table 3). This could suggest that the fluorescence change upon PcTX1 application reflects the transition of the channels from a closed to a SSD state upon PCTX1 application. However, this hypothesis is contradicted by several observations. First, during the washout of PcTX1 in K105C* or V80C*, the sustained fluorescence signal indicates a long-lived conformational change, but the channels can already be fully activated even before the fluorescence signal fully returns to baseline (Figure 1E, G and Figure 2A). Secondly, in the $\mathrm{K} 105 \mathrm{C} * \mathrm{~F} 350 \mathrm{~L}$ and $\mathrm{V} 80 \mathrm{C} * \mathrm{~F} 350 \mathrm{~L}$ variants, PcTx1 still introduces comparable conformational changes as seen for K105C* or V80C* respectively, yet no functional inhibition could be seen (Figure 3), making it unlikely that channels have ever entered SSD. We therefore favour the notion that labelling allows us to track conformational changes that do not simply report on a known functional transition, but rather reflect unique ECD conformational alterations induced by PcTx1.

\section{Identification of three distinct modes of action for PcTX1}

Our data suggest the presence of three distinct modes of action for PcTx1: a 'Loose', a tight 'Global', and a tight 'ECDonly'. We propose PCTX1 to bind loosely to the ASIC1a high-pH closed state that remains in the resting conformation even in the presence of PcTX1. This association does not result in an ECD conformational change, possibly due to the acidic pocket remaining in an expanded configuration (Yoder, Yoshioka et al. 2018), but its physical nature is evident from the slight but consistent downward deflection in the fluorescence signal observed with PCTX1 application at pH 8.0, as well as the fact that it increases the proton sensitivity of the channel (Figure 2B left, middle). Importantly, this association of PcTX1 is readily reversible, as long as the channel remains in a closed state, possibly as a result of unfavorable conformational and/or electrostatic 
interactions. In the tight 'Global' mode, PCTX1 induces an alteration of the ECD conformation and also inhibits ion flux, likely through tight coupling to the channel pore, and thereby leads to functional inhibition of ASIC1a. Interestingly, an earlier study reported prolonged functional effects of PcTx1 even after washout (Escoubas, De Weille et al. 2000), possibly indicating that the 'Global' binding mode can be long-lived. However, we further observe conformational ECD changes that last substantially beyond the timeframe of observable functional effects on the channel pore. This long-lasting 'ECD only' binding mode has not been characterized before and we cannot ultimately determine if it is elicited through PcTX1 molecules bound in a functionally non-productive conformation or through diffusion of PcTx1 molecules that were previously membrane-associated (Gupta, Zamanian et al. 2015). However, the potential physiological implications of this long-lasting conformational state are evident from our observation that it affects the activity of the endogenous neuropeptide BigDyn. And since increased BigDyn levels have been linked to acidosis-induced neuronal cell death (Sherwood and Askwith 2009), this long-lasting interference of the 'ECD only' only conformation on the ASIC-BigDyn interaction could contribute to neuroprotective effects and provide a conceptually new route for ASIC-targeting therapeutics. Interestingly, such functionally silent states and conformations have previously been suggested to occur also in other ligand-gated ion channels (Dahan, Dibas et al. 2004, Pless, Dibas et al. 2007, Pless and Lynch 2009, Munro, Ladefoged et al. 2019) and could, at least in theory, be used to modulate protein-ligand and proteinprotein interactions. We further speculate that long-lasting conformational changes could also interfere with recently proposed metabotropic functions of ASIC1a by altering intracellular protein-protein interactions (Wang, Wang et al. 2015, Wang, Liu et al. 2020), although this would require additional verification.

\section{The F350L mutation destabilizes a PCTX1-induced conformational states}

Consistent with previous work (Sherwood, Franke et al. 2009, Saez, Deplazes et al. 2015), we observed that although the F350L mutation shifts the PCTX1 IC 50 by about three orders of magnitude, ASIC1a is not rendered completely insensitive towards PcTx1 modulation. Reduced PcTx1-inhibition could, in part, result from the reduced $\mathrm{pH}$ sensitivity of the $\mathrm{F} 350 \mathrm{~L}$ variant, in line with the critical role of $\mathrm{pH}$ on PcTx1 modulation observed for human and rat ASIC1a (Cristofori-Armstrong, Saez et al. 2019). A mutant whose $\mathrm{pH}_{50}$ is shifted towards higher proton concentrations (right-shifted) would show less inhibition even if PcTx1 sensitivity was maintained. However, our VCF experiments rule out decreased $\mathrm{pH}$ sensitivity as the main cause for reduced PcTx1 sensitivity since we corrected for the shifts in SSD. Applied at pH 7.4, 300 nM PcTx1 (below the IC 50 of the unlabelled F350L variant) does not lead to conformational changes, but does so at pH 7.3, although no functional effects are observed. These experiments indicate that PcTX1 interacts with F350L* and introduces conformational changes, despite the lack of functional consequences at these concentrations. This is in line with earlier results that suggested for PCTX1 to still bind to F352L hASIC1a, as pre-incubation with PcTX1 prevented the modulatory effects of BigDyn (Sherwood and Askwith 2009) and PcTx1 could still be crosslinked with the mutant channel (Braun, Friis et al. 2021). Similarly, experiments from ASIC1a/2a concatemers of different subunit stoichiometries suggested that PCTX1 also binds to ASIC1a-2a interfaces (Joeres, Augustinowski et al. 2016), despite the fact that ASIC2a subunits contain a Leu at the position corresponding to 350 in mASIC1a. Hence, the F350L mutation seems to diminish functional modulation by PcTx1, but not necessarily binding. Indeed, our fluorescence data indicate that the F350L mutation appears to destabilize the 
PcTx1-induced states, leading to faster return of the fluorescence signal to baseline levels, suggestive of a closed apo state.

\section{Stoichiometry of toxin binding}

Structural data have recently provided a wealth of insight into the molecular details of where and how drugs or toxins binding to ion channels. Yet these data cannot directly inform on the ligand-receptor stoichiometry required for the full functional effect. For example, cryo-EM structures of homotetrameric KCNQ channels suggest a stoichiometric binding of the antiepileptic small-molecule drug retigabine (i.e. one drug per each of the four subunits), while functional work shows that binding of a single retigabine molecule is sufficient to elicit the full functional effect of the channel activator (Yau, Kim et al. 2018, Li, Wu et al. 2021, Li, Zhang et al. 2021). Similarly, binding of a single alpha-bungarotoxin molecule to the heteromeric nAChR pentamer is sufficient for channel inhibition, despite two molecules being present in a cryo-EM co-structure (daCosta, Free et al. 2015, Rahman, Teng et al. 2020).

Here, we show that concatemers with homomeric subunit composition display comparable $\mathrm{pH}$ sensitivities for activation and SSD, along with similar sensitivity to PCTX1 compared to their homotrimeric counterparts. Functionally, we demonstrate that two WT ASIC1a subunits are sufficient for WT-like PCTX1 sensitivity at physiological pH. Our VCF results, however, revealed that a single F350L subunit is sufficient to destabilize the PCTX1-induced ECD conformation, which then further decreases in channels containing two or three mutant subunits. Together, the proposed binding modes emphasize that the conformational changes of the ECD are partially decoupled from the TMD: interfering with the binding of a single PcTx1 molecule is sufficient to alter the stability of the ECD conformation, while the pore (or the coupling to it) translates to closed channels only if binding of two or more PcTx1 molecules is possible (Figure 6).

\section{Conclusions}

The conformational landscape of ion channels is likely to far exceed the conformations uncovered by structural or functional studies, as it is often challenging to capture such states in compelling ways. By combining concatemeric construct design with voltage-clamp fluorometry, we provide insight into functionally silent conformational changes of the mASIC1a ECD during PCTX1 modulation and assess the contribution of individual subunits to the overall conformational state and on the functional state of the pore. We found that PcTx1 can bind to mASIC1a with at least three distinct binding modes. In addition, PcTx1 induces a long-lived conformational state in the ASIC1a ECD that is uncoupled from the pore but alters ASIC1a pharmacology. Lastly, the work presented here, to our knowledge, constitutes the first use of concatemeric multisubunit assemblies engineered for VCF recordings, thus offering the possibility to label a defined subset of subunits to unveil potential asymmetric gating and binding events, such as those proposed for a variety of ligand-gated ion channels (Jasti, Furukawa et al. 2007, Baconguis and Gouaux 2012, Mowrey, Cheng et al. 2013, Zhu, Noviello et al. 2018, Guros, Balijepalli et al. 2020, Zhang, Dijkman et al. 2021). 
bioRxiv preprint doi: https://doi.org/10.1101/2021.06.21.449215; this version posted August 26, 2021. The copyright holder for this preprint (which was not certified by peer review) is the author/funder. All rights reserved. No reuse allowed without permission.

\section{Acknowledgments}

We acknowledge funding from the Lundbeck Foundation (R303-2018-3030 to SAH and R313-2019-571 to SAP), the Brødrene Hartmanns Fond, and the European Union's Horizon 2020 research and innovation program under the Marie Skłodowska-Curie grant agreement No 834274 (to SAH). We thank Dr. Han Chow Chua for comments on the manuscript. 


\section{Materials and Methods}

\section{Molecular biology.}

The complementary DNA (cDNA) encoding mouse ASIC1a (mASIC1a) was used as previously described (Lynagh, Flood et al. 2017). Site-directed mutagenesis was performed using PfuUltrall Fusion polymerase (Agilent) and custom DNA mutagenesis primers (Eurofins Genomics). All sequences were confirmed by sequencing of the full coding frame (Eurofins Genomics or Macrogen). cDNAs were linearized with EcoRI and capped cRNA was transcribed with the Ambion mMESSAGE mMACHINE SP6 kit (Thermo Fisher Scientific).

\section{Concatemer design}

Mouse ASIC1a concatemeric constructs were generated as described before (Lynagh, Flood et al. 2017). Briefly, the concatemeric mASIC1a constructs were generated by cloning out the mASIC1a insert from the pSP64 vector using forward and reverse primers containing additional HindIII and Sall, Sall and BamHI or BamHI and Sacl sequences, respectively (Figure S2). This generated three distinct inserts, $a, b$ and $c$, which were gelpurified using the Gel/PCR DNA fragment kit (Geneaid). The inserts were digested with their respective restriction enzymes, insert $a$ : HindIII and Sall, insert b: Sall and BamHI, and insert c: BamHI and Sacl. Insert $a$ was ligated into the empty pSP64 vector (Promega) double-digested with HindIII and Sall. The resulting vector was then double-digested with Sall and BamHI to insert segment $b$ in similar way. The $a-b$ plasmid was then double-digested using BamHI and Sacl and segment $c$ was ligated into it. This yielded the final " $a-b-c$ " mASIC1a concatemer. From this construct, WT inserts were replaced by mASIC1a inserts containing amino acid substitutions generated using the same primers and restriction sites. Full concatemer sequences were confirmed using primers recognizing the unique restriction sites.

Electrophysiological recordings. Oocytes were surgically removed from adult female Xenopus laevis and prepared as previously described (Lynagh, Flood et al. 2017). Oocytes were injected with 0.1 to $10 \mathrm{ng}$ cRNA of mASIC1a (volumes between 9 and $50 \mathrm{~nL}$ ) and incubated for $1-4$ days at $18{ }^{\circ} \mathrm{C}$ in Leibovitz's L-15 medium (Gibco), supplemented with $3 \mathrm{mM} \mathrm{L-glutamine,} 2.5 \mathrm{mg} / \mathrm{ml}$ gentamycin and $15 \mathrm{mM} \mathrm{HEPES}$ adjusted to $\mathrm{pH}$ 7.6. Typically, larger amounts of mutant mASIC1a cRNA were injected compared to WT mASIC1a. For electrophysiological recordings, oocytes were transferred to a recording chamber (Dahan, Dibas et al. 2004), continuously perfused ( $2.5 \mathrm{~mL} / \mathrm{min}$ ) with buffer containing (in $\mathrm{mM}$ ): $96 \mathrm{NaCl}, 2 \mathrm{KCl}, 1.8 \mathrm{BaCl}_{2}, 2 \mathrm{MgCl}$, and 5 HEPES, pH adjusted by $\mathrm{NaOH}$ or $\mathrm{HCl}$. Solutions exchange was achieved using a gravity-driven 8-line automated perfusion system operated by a ValveBank module (AutoMate Scientific). In experiments where PcTx1 was used, all solutions were supplemented with $0.05 \%$ bovine serum albumin ( $\geq 98 \%$ essentially fatty acid-free, Sigma Aldrich). Currents were recorded using microelectrodes (borosilicate capillaries $1.2 \mathrm{~mm}$ OD, $0.94 \mathrm{~mm}$ ID, Harvard Apparatus), backfilled with $3 \mathrm{M} \mathrm{KCl}(0.3-1.5 \mathrm{M} \Omega$ ) and an OC-725C amplifier (Warner Instruments). The current signal was acquired at $500 \mathrm{~Hz}$, filtered by a 50-60 Hz noise eliminator (Hum Bug, Quest Scientific) and digitized using an Axon ${ }^{\mathrm{TM}}$ Digidata 1550 Data Acquisition System and the pClamp (10.5.1.0) software (Molecular Devices). The current signal was further digitally filtered at $2.8 \mathrm{~Hz}$ using an 8-pole Bessel low-pass filter prior to analysis. Displayed current traces have been subjected to additional 50x data reduction.

Synthetic PcTx1 was obtained from Alomone Labs (>95\% purity). BigDyn was synthesized using automated solid-phase peptide synthesis, followed by purification via reversed-phase high-performance liquid 
chromatography (RP-HPLC) as described previously (Borg, Braun et al. 2020). Peptide stock solutions were prepared in MilliQ (18.2 $\mathrm{M} \Omega$ resistivity) and stored at $-20^{\circ} \mathrm{C}$. Prior to recording, the peptide was diluted to the desired concentration in recording solution.

Concentration-response relationships of MASIC1a activation were determined by $20 \mathrm{~s}$ applications of solutions with decreasing $\mathrm{pH}$-values. Between each $20 \mathrm{~s}$ application, the oocytes were left to recover for at least $1 \mathrm{~min}$ in $\mathrm{pH} 7.4$ solution (unless stated otherwise). The currents elicited by the $20 \mathrm{~s}$ application of acidic $\mathrm{pH}$ were normalized to the largest current size for each of individual oocyte tested. Steady-state desensitization (SSD) concentration-response relationships were determined by exposing ASIC1a expressing oocytes to a $20 \mathrm{~s}$ application of $\mathrm{pH} 5.6$ (unless stated otherwise), while decreasing the resting $\mathrm{pH}$ in between applications of $\mathrm{pH}$ 5.6. In order to ensure that the observed current desensitization was not due to general current run-down, a final application of $\mathrm{pH} 5.6$ was performed after a 2 min resting period at a resting $\mathrm{pH}$ that resulted in saturating current responses. Traces were used for further analysis only if the final pH 5.6 application resulted in currents that were $\geq 80 \%$ of the same resting pH prior to the SSD protocol. For testing of the effect of PcTx1 on the ASIC1a current response, channels were activated by $20 \mathrm{~s}$ application of $\mathrm{pH}$ 5.6. Hereafter the $\mathrm{pH}$ solution returned to resting $\mathrm{pH}$ to allow channel recovery. PcTx1 was then applied at the desired pH for 1 min prior to a 20 s application of $\mathrm{pH} 5.6$.

Voltage-clamp fluorometry. For voltage-clamp fluorometry (VCF), 5-20 ng of mASIC1a mRNA or 50 ng for concatemeric constructs were injected into oocytes which were then incubated for 2-7 days. On the day of the recording, oocytes were labeled by incubating them for 30 min with $10 \mu \mathrm{M}$ AlexaFlour $488 \mathrm{C} 5$-maleimide (Thermo Fisher Scientific) at room temperature in OR2 solution containing (in $\mathrm{mM}$ ) $82.5 \mathrm{NaCl}, 2 \mathrm{KCl}, 1 \mathrm{MgCl}$, $\mathrm{pH} 7.4$, subsequently washed twice with OR2, and stored in the dark until further use. Concatemeric constructs were kept in the dark for several hours, as it tended to reduce background fluorescence. VCF recordings were done as previously described (Borg, Braun et al. 2020) using an inverse microscope (IX73 with LUMPlanFL N 40x, Olympus) an Olympus TH4-200 halogen lamp as light source and using a standard GFP filter-set (Olympus). The emission was detected using a P25PC-19 photomultiplier tube (Sens-Tech) and photon-to-voltage converter (IonOptix). Current and fluorescence signals were acquired, filtered, digitized and digitally filtered as described for the other electrophysiology recordings.

For SSD and pH-dependent fluorescence measurements, running buffer at pH 7.6 was used. Labelled channels were activated for $20 \mathrm{~s}$ using pH 6.0, washed for $60 \mathrm{~s}$ with pH 7.6 buffer, exposed for $60 \mathrm{~s}$ to buffer at conditioning $\mathrm{pH}$ and then directly activated again (Figure S1A). Control activations at pH 6.0 were conducted between each SSD measurement. For PcTx1 responses, running buffer at $0.3 \mathrm{pH}$ units above the established $\mathrm{pH}_{50}$ fluorescence was used, unless stated otherwise. First channels were activated for $20 \mathrm{~s}$ with pH 5.5, washed for $60 \mathrm{~s}$ with running buffer before applying $300 \mathrm{nM}$ PcTx1. Washout protocols either included 3 min exposure to running buffer, pH 8.4 buffer, or a mix of the two (20 s running buffer, $60 \mathrm{~s} \mathrm{pH} \mathrm{8.4,} 40$ s running buffer, 60s $\mathrm{pH}$ 8.4). In experiments where PcTx1 was applied at pH 8.0 for 40 s, channels were subsequently either directly exposed to pH 7.4 or activated first with $\mathrm{pH} 5.5$ for $20 \mathrm{~s}$, or pH 8.0 for $40 \mathrm{~s}$ before switching to $\mathrm{pH} 7.4$. Fluorescence levels $60 \mathrm{~s}$ after switching to $\mathrm{pH} 7.4$ were compared to the levels reached during activation with pH 5.5. For BigDyn experiments, 1 MM BigDyn was applied at pH 7.4 either for $60 \mathrm{~s}$ before PcTx 1 application, or for $120 \mathrm{~s}$ once $300 \mathrm{nM}$ PcTx1 had been washed off with pH 7.4 for $120 \mathrm{~s}$. 
Surface protein purification. Oocyte surface proteins were purified using the Pierce Cell Surface Protein Isolation Kit (Thermo Fisher Scientific), modified here for oocytes as previously described (Lynagh, Flood et al. 2017). In brief, 30 oocytes were injected with RNA of a single mASIC1a construct. After two days of incubation the oocytes were washed with phosphate buffered saline (PBS) and incubated for $25 \mathrm{~min}$ at room temperature with sulfo-NHS-SS-biotin. Hereafter, the reaction was quenched and oocytes were washed with PBS. The oocytes were lysed, centrifuged, and the clarified supernatant was transferred to a spin column containing equilibrated NeutrAvidin Agarose and incubated for 1 hour at room temperature under gentle agitation. The flow-through was discarded and the NeutrAvidin Agarose was washed and centrifuged. The wash procedure was repeated for a total of three times. Prior to elution, $200 \mu \mathrm{L}$ SDS-PAGE sample buffer containing $50 \mathrm{mM}$ dithiothreitol (DTT) was added and the column was incubated for $60 \mathrm{~min}$ at room temperature under agitation. The protein was then eluted by centrifugation. The concentration of protein was determined using the NanoDrop One $e^{\mathrm{C}} \mathrm{UV}$-Vis spectrophotometer and the samples were stored at $-20^{\circ} \mathrm{C}$.

Western blotting. Oocyte surface protein samples were denatured for $5 \mathrm{~min}$ at $90{ }^{\circ} \mathrm{C}$ in LDS sample buffer containing reducing agent (50 mM DTT) (NuPAGE ${ }^{\circ}$, Thermo Fisher Scientific). Protein samples (10-120 $\mu \mathrm{g}$, dependent on the construct in order to get approximately equal band intensity), Novex Sharp pre-stained standard (Thermo Fisher Scientific) were separated by SDS-PAGE using a 4-12 \% Bis-Tris protein gel (NuPAGE*, Thermo Fisher Scientific). Protein bands were transferred to a polyvinylidene difluoride (PVDF) membrane $\left(0.45 \mu \mathrm{m}\right.$, Thermo Fisher Scientific) in transfer buffer (NuPAGE ${ }^{\circ}$, Thermo Fisher) containing $10 \%$ methanol $(\mathrm{MeOH})$. After transfer, the PVDF membrane was rinsed with tris buffered saline $0.1 \%$ tween (TBST) and subsequently blocked with $0.5 \%$ non-fat dried milk (AppliChem) in TBST using the Snap i.d. 2.0 Protein Detection System (Millipore). The blot was then incubated for $10 \mathrm{~min}$ at room temperature with a 1:1000 dilution of rabbit polyclonal anti-ASIC1a antibody (OSR00097W, Thermo Fisher Scientific) in blocking buffer followed by $10 \mathrm{~min}$ incubation at room temperature with goat anti-rabbit IgG/horseradish peroxidaseconjugate (1:10,000 dilution of $1 \mathrm{mg} / \mathrm{mL}$ stock in blocking buffer) (A16110, Thermo Fisher Scientific). The blot was developed using enhanced chemoluminescence detection reagents (Pierce ${ }^{\mathrm{TM}} \mathrm{ECL}$ Western Blotting Substrate, Thermo Fisher Scientific) and visualized using a PXi Touch Gel Imaging System (Syngene).

Data analysis. Data analyses were performed in Prism (8.0) (GraphPad Software). Fluorescence baseline was adjusted for all fluorescence traces before analysis. For proton concentration-response data, peak amplitudes were normalized to control activations, plotted as a function of $\mathrm{pH}$, and fitted with the Hill equation constrained at $\min =0$ (and $\max =1$ for TEVC data) for each recording. The resulting $E C_{50}$ and Hill values were averaged to give the reported means $\pm 95 \mathrm{Cl}$ in the tables. For PcTx1 inhibition experiments, current responses after PcTx1 treatment were normalized to the current responses without PcTx1 incubation for each oocyte. The average data for the different PCTX1 concentrations were fitted using a Hill-equation constrained at $\min =0$ and $\max =$ 1 . For display in figures, a single fit to the average normalized responses $( \pm 95 \mathrm{Cl})$ is shown. The change in fluorescence upon PcTx1 application was normalized to the neighbouring pH 5.5 application. Washout of PcTx1 in VCF experiments was reported as the level of fluorescence under running buffer at the end of the $3 \mathrm{~min}$ 
washout relative to the PcTx1-induce fluorescence change. Fluorescence signal intensity was reported as percentage change relative to the baseline fluorescence level.

All bar diagrams and summarized data points are presented as mean $\pm 95 \mathrm{Cl}$ unless stated otherwise and number of replicates ( $n$ ) represent individual experimental oocytes. Results were obtained from at least two batches of oocytes. An unpaired t-Mann-Whitney test was used to compare two groups. Multiple comparisons in Figure S1E and F were made with one-way analysis of variance with Dunnett's comparison to a control value (i.e. washout using running buffer). A significance level of $p<0.05$ was applied for all analyses. All graphs and illustrations were made in Prism (8.0) (GraphPad Software) and Illustrator CC (Adobe), structure rendering was done using ChimeraX (Pettersen, Goddard et al. 2021). 


\section{References}

Baconguis, I., C. J. Bohlen, A. Goehring, D. Julius and E. Gouaux (2014). "X-ray structure of acid-sensing ion channel 1-snake toxin complex reveals open state of a Na(+)-selective channel." Cell 156(4): 717-729.

Baconguis, I. and E. Gouaux (2012). "Structural plasticity and dynamic selectivity of acid-sensing ion channelspider toxin complexes." Nature 489(7416): 400-405.

Borg, C. B., N. Braun, S. A. Heusser, Y. Bay, D. Weis, I. Galleano, C. Lund, W. Tian, L. M. Haugaard-Kedstrom, E. P. Bennett, T. Lynagh, K. Stromgaard, J. Andersen and S. A. Pless (2020). "Mechanism and site of action of big dynorphin on ASIC1a." Proc Natl Acad Sci U S A 117(13): 7447-7454.

Braun, N., S. Friis, C. Ihling, A. Sinz, J. Andersen and S. A. Pless (2021). "High-throughput characterization of photocrosslinker-bearing ion channel variants to map residues critical for function and pharmacology." bioRxiv: 2020.2011.2024.392498.

Chassagnon, I. R., C. A. McCarthy, Y. K. Chin, S. S. Pineda, A. Keramidas, M. Mobli, V. Pham, T. M. De Silva, J. W. Lynch, R. E. Widdop, L. D. Rash and G. F. King (2017). "Potent neuroprotection after stroke afforded by a double-knot spider-venom peptide that inhibits acid-sensing ion channel 1a." Proc Natl Acad Sci U S A 114(14): 3750-3755.

Chen, X., H. Kalbacher and S. Grunder (2005). "The tarantula toxin psalmotoxin 1 inhibits acid-sensing ion channel (ASIC) 1a by increasing its apparent H+ affinity." J Gen Physiol 126(1): 71-79.

Chen, X., H. Kalbacher and S. Grunder (2006). "Interaction of acid-sensing ion channel (ASIC) 1 with the tarantula toxin psalmotoxin 1 is state dependent." J Gen Physiol 127(3): 267-276.

Cristofori-Armstrong, B. and L. D. Rash (2017). "Acid-sensing ion channel (ASIC) structure and function: Insights from spider, snake and sea anemone venoms." Neuropharmacology 127: 173-184.

Cristofori-Armstrong, B., N. J. Saez, I. R. Chassagnon, G. F. King and L. D. Rash (2019). "The modulation of acidsensing ion channel 1 by PCTX1 is $\mathrm{pH}$-, subtype- and species-dependent: Importance of interactions at the channel subunit interface and potential for engineering selective analogues." Biochem Pharmacol 163: 381390.

daCosta, C. J. B., C. R. Free and S. M. Sine (2015). "Stoichiometry for $\alpha$-bungarotoxin block of $\alpha 7$ acetylcholine receptors." Nat Commun 6(1): 8057.

Dahan, D. S., M. I. Dibas, E. J. Petersson, V. C. Auyeung, B. Chanda, F. Bezanilla, D. A. Dougherty and H. A. Lester (2004). "A fluorophore attached to nicotinic acetylcholine receptor beta M2 detects productive binding of agonist to the alpha delta site." Proc Natl Acad Sci U S A 101(27): 10195-10200.

Dawson, R. J., J. Benz, P. Stohler, T. Tetaz, C. Joseph, S. Huber, G. Schmid, D. Hugin, P. Pflimlin, G. Trube, M. G. Rudolph, M. Hennig and A. Ruf (2012). "Structure of the acid-sensing ion channel 1 in complex with the gating modifier Psalmotoxin 1." Nat Commun 3: 936.

Du, J., L. R. Reznikov, M. P. Price, X.-m. Zha, Y. Lu, T. O. Moninger, J. A. Wemmie and M. J. Welsh (2014). "Protons are a neurotransmitter that regulates synaptic plasticity in the lateral amygdala." Proceedings of the National Academy of Sciences of the United States of America 111(24): 8961-8966.

Escoubas, P., J. R. De Weille, A. Lecoq, S. Diochot, R. Waldmann, G. Champigny, D. Moinier, A. Menez and M. Lazdunski (2000). "Isolation of a tarantula toxin specific for a class of proton-gated $\mathrm{Na}$ + channels." J Biol Chem 275(33): 25116-25121.

Gründer, S. (2020). Acid-Sensing Ion Channels The Oxford Handbook of Neuronal Ion Channels. A. Bhattacharjee, Oxford University Press: 1-56.

Gupta, K., M. Zamanian, C. Bae, M. Milescu, D. Krepkiy, D. C. Tilley, J. T. Sack, V. Yarov-Yarovoy, J. I. Kim and K. J. Swartz (2015). "Tarantula toxins use common surfaces for interacting with Kv and ASIC ion channels." Elife 4. 
bioRxiv preprint doi: https://doi.org/10.1101/2021.06.21.449215; this version posted August 26, 2021. The copyright holder for this preprint (which was not certified by peer review) is the author/funder. All rights reserved. No reuse allowed without permission.

Guros, N. B., A. Balijepalli and J. B. Klauda (2020). "Microsecond-timescale simulations suggest 5-HT-mediated preactivation of the 5-HT3A serotonin receptor." Proc Natl Acad Sci U SA 117(1): 405-414.

Jasti, J., H. Furukawa, E. B. Gonzales and E. Gouaux (2007). "Structure of acid-sensing ion channel 1 at 1.9 A resolution and low pH." Nature 449(7160): 316-323.

Joeres, N., K. Augustinowski, A. Neuhof, M. Assmann and S. Grunder (2016). "Functional and pharmacological characterization of two different ASIC1a/2a heteromers reveals their sensitivity to the spider toxin PcTx1." Sci Rep 6: 27647.

Kellenberger, S. and L. Schild (2015). "International Union of Basic and Clinical Pharmacology. XCI. structure, function, and pharmacology of acid-sensing ion channels and the epithelial Na+ channel." Pharmacol Rev 67(1): 1-35.

Li, T., K. Wu, Z. L. Yue, Y. F. Wang, F. Zhang and H. Z. Shen (2021). "Structural Basis for the Modulation of Human KCNQ4 by Small-Molecule Drugs." Molecular Cell 81(1): 25-+.

Li, X. X., Q. S. Zhang, P. P. Guo, J. Fu, L. H. Mei, D. S. Lv, J. Q. Wang, D. W. Lai, S. Ye, H. Y. Yang and J. T. Guo (2021). "Molecular basis for ligand activation of the human KCNQ2 channel." Cell Research 31(1): 52-61.

Lynagh, T., E. Flood, C. Boiteux, M. Wulf, V. V. Komnatnyy, J. M. Colding, T. W. Allen and S. A. Pless (2017). "A selectivity filter at the intracellular end of the acid-sensing ion channel pore." Elife 6.

Mowrey, D., M. H. Cheng, L. T. Liu, D. Willenbring, X. Lu, T. Wymore, Y. Xu and P. Tang (2013). "Asymmetric ligand binding facilitates conformational transitions in pentameric ligand-gated ion channels." $\_$Am Chem Soc 135(6): 2172-2180.

Munro, L., L. K. Ladefoged, V. Padmanathan, S. Andersen, B. Schiott and A. S. Kristensen (2019). "Conformational Changes in the 5-HT3A Receptor Extracellular Domain Measured by Voltage-Clamp Fluorometry." Mol Pharmacol 96(6): 720-734.

Pettersen, E. F., T. D. Goddard, C. C. Huang, E. C. Meng, G. S. Couch, T. I. Croll, J. H. Morris and T. E. Ferrin (2021). "UCSF ChimeraX: Structure visualization for researchers, educators, and developers." Protein Sci 30(1): 70-82.

Pless, S. A., M. I. Dibas, H. A. Lester and J. W. Lynch (2007). "Conformational variability of the glycine receptor M2 domain in response to activation by different agonists." J Biol Chem 282(49): 36057-36067.

Pless, S. A. and J. W. Lynch (2009). "Ligand-specific conformational changes in the alpha1 glycine receptor ligand-binding domain." J Biol Chem 284(23): 15847-15856.

Qiang, M., X. Dong, Z. Zha, X. K. Zuo, X. L. Song, L. Zhao, C. Yuan, C. Huang, P. Tao, Q. Hu, W. G. Li, W. Hu, J. Li, Y. Nie, D. Buratto, F. Zonta, P. Ma, Z. Yu, L. Liu, Y. Zhang, B. Yang, J. Xie, T. L. Xu, Z. Qu, G. Yang and R. A. Lerner (2018). "Selection of an ASIC1a-blocking combinatorial antibody that protects cells from ischemic death." Proc Natl Acad Sci U S A 115(32): E7469-E7477.

Rahman, M. M., J. Teng, B. T. Worrell, C. M. Noviello, M. Lee, A. Karlin, M. H. B. Stowell and R. E. Hibbs (2020). "Structure of the Native Muscle-type Nicotinic Receptor and Inhibition by Snake Venom Toxins." Neuron 106(6): 952-962.e955.

Saez, N. J., E. Deplazes, B. Cristofori-Armstrong, I. R. Chassagnon, X. Lin, M. Mobli, A. E. Mark, L. D. Rash and G. F. King (2015). "Molecular dynamics and functional studies define a hot spot of crystal contacts essential for PcTx1 inhibition of acid-sensing ion channel 1a." Br J Pharmacol 172(20): 4985-4995.

Sherwood, T., R. Franke, S. Conneely, J. Joyner, P. Arumugan and C. Askwith (2009). "Identification of protein domains that control proton and calcium sensitivity of ASIC1a." J Biol Chem 284(41): 27899-27907.

Sherwood, T. W. and C. C. Askwith (2009). "Dynorphin opioid peptides enhance acid-sensing ion channel 1a activity and acidosis-induced neuronal death." J Neurosci 29(45): 14371-14380. 
Sherwood, T. W., K. G. Lee, M. G. Gormley and C. C. Askwith (2011). "Heteromeric acid-sensing ion channels (ASICs) composed of ASIC2b and ASIC1a display novel channel properties and contribute to acidosis-induced neuronal death." J Neurosci 31(26): 9723-9734.

Vullo, S., G. Bonifacio, S. Roy, N. Johner, S. Berneche and S. Kellenberger (2017). "Conformational dynamics and role of the acidic pocket in ASIC pH-dependent gating." Proc Natl Acad Sci U S A 114(14): 3768-3773.

Wang, J. J., F. Liu, F. Yang, Y. Z. Wang, X. Qi, Y. Li, Q. Hu, M. X. Zhu and T. L. Xu (2020). "Disruption of autoinhibition underlies conformational signaling of ASIC1a to induce neuronal necroptosis." Nat Commun 11(1): 475.

Wang, Y. Z., J. J. Wang, Y. Huang, F. Liu, W. Z. Zeng, Y. Li, Z. G. Xiong, M. X. Zhu and T. L. Xu (2015). "Tissue acidosis induces neuronal necroptosis via ASIC1a channel independent of its ionic conduction." Elife 4.

Wemmie, J. A., J. Chen, C. C. Askwith, A. M. Hruska-Hageman, M. P. Price, B. C. Nolan, P. G. Yoder, E. Lamani, T. Hoshi, J. H. Freeman, Jr. and M. J. Welsh (2002). "The acid-activated ion channel ASIC contributes to synaptic plasticity, learning, and memory." Neuron 34(3): 463-477.

Wemmie, J. A., R. J. Taugher and C. J. Kreple (2013). "Acid-sensing ion channels in pain and disease." Nat Rev Neurosci 14(7): 461-471.

Wu, Y., Z. Chen and C. M. Canessa (2019). "A valve-like mechanism controls desensitization of functional mammalian isoforms of acid-sensing ion channels." Elife 8.

Xiong, Z. G., X. M. Zhu, X. P. Chu, M. Minami, J. Hey, W. L. Wei, J. F. MacDonald, J. A. Wemmie, M. P. Price, M. J. Welsh and R. P. Simon (2004). "Neuroprotection in ischemia: blocking calcium-permeable acid-sensing ion channels." Cell 118(6): 687-698.

Yau, M. C., R. Y. Kim, C. K. Wang, J. R. Li, T. Ammar, R. Y. Yang, S. A. Pless and H. T. Kurata (2018). "One drugsensitive subunit is sufficient for a near-maximal retigabine effect in KCNQ channels." Journal of General Physiology 150(10): 1421-1431.

Yoder, N., C. Yoshioka and E. Gouaux (2018). "Gating mechanisms of acid-sensing ion channels." Nature 555(7696): 397-401.

Zhang, Y., P. M. Dijkman, R. Zou, M. Zandl-Lang, R. M. Sanchez, L. Eckhardt-Strelau, H. Kofeler, H. Vogel, S. Yuan and M. Kudryashev (2021). "Asymmetric opening of the homopentameric 5-HT3A serotonin receptor in lipid bilayers." Nat Commun 12(1): 1074.

Zhu, S., C. M. Noviello, J. Teng, R. M. Walsh, Jr., J. J. Kim and R. E. Hibbs (2018). "Structure of a human synaptic GABAA receptor." Nature 559(7712): 67-72. 